\title{
Simplified Computation of Electromagnetic Band-Gap Properties of Via-Holed Metal Patches
}

\author{
K. Rambabu \\ Department of Electrical and Computer Engineering \\ University of Alberta, Edmonton, Alberta, Canada
}

\author{
M. Mokhtaari, J. Bornemann \\ Department of Electrical and Computer Engineering \\ University of Victoria, Victoria, BC, Canada
}

\begin{abstract}
A simple analytical method for the analysis and design of electromagnetic band-gap (EBG) structures formed by viaholed metal patches is presented. Contrary to a known approach, this technique correctly predicts the first stopband, which is often utilized in printed antenna applications, and significantly extends the frequency range of analysis by including complex propagation constants. The approach is verified by comparisons with results of commercially available software.
\end{abstract}

Keywords - Electromagnetic band-gap structures; transmission line theory.

\section{INTRODUCTION}

Advances and applications of Electromagnetic Band-Gap (EBG) structures have shown that the excitation of surface waves can be considerably reduced. Several types of microstrip-based EBG structures have been proposed for a variety of applications, e.g. [1], and were analyzed and designed by using numerical techniques such as the finitedifference time-domain [2] and the finite-element method [3]. Equivalent-circuit methods have been developed in order to quickly design EBG structures for desired pass- and stopbands. These models can be easily programmed when field solvers (such as [2]) are not available. Moreover, they require less computation time and, therefore, aid in designing EBG's in a timely fashion [4], [5], [6].

One of these techniques was presented in [5] for applications to via-holed patches, e.g. [7], [8], as shown in Fig. 1. In applying the related formulation, however, it was found that the method introduces certain assumptions, which prevent the computation of the first stopband, i.e. the frequency range between d.c. and the first passband. Moreover, the technique in [5], as well as those in [4] and [6], assume non-attenuated propagation in the passband and, therefore, fail to allow simultaneous propagation and attenuation.

Since in the first stopband, miniaturization is achieved, i.e. the size of the EBG is small, thus offering attractive solutions towards compact component designs, this paper introduces an extended analytical method with the goal to develop a simple yet accurate computational analysis and design tool for EBG's over a broad bandwidth. Based on approaches, which differ from those in the references, to model the gaps between microstrip patches in both directions (Fig. 1), this technique extends the applicable frequency range by correctly predicting the first stopband. Moreover, the model is applicable over a wider bandwidth due to the general assumption of a complex propagation constant. Reasonable agreement is demonstrated with results obtained from commercial field solvers as far as attenuation over a limited number of cells is concerned.

\section{THEORY}

Fig. 1 shows the schematic diagram of the EBG lattice and its essential dimensions. A square metal sheet connected to the continuous ground plane through a centered via hole or thin wire [7] constitutes the unit cell of the lattice.

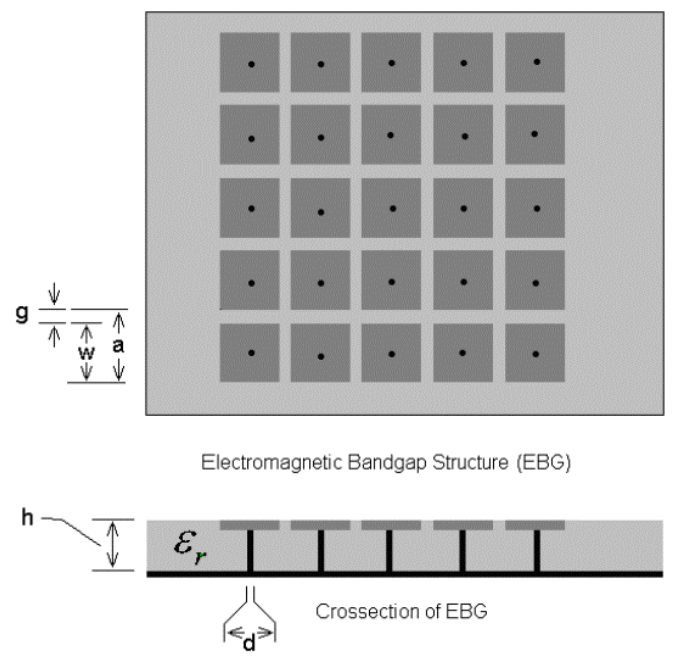

Figure 1. Schematic diagram of the EBG structure formed by metal patches with centered via holes.

Our model is shown in Fig. 2. Fig. 2a shows the cross section in longitudinal direction. It includes the microstrip line of impedance $Z_{0}$ and propagation constant $\beta_{m}$, the inductance $L$ of the via hole, and the capacitive section to represent the gap between patches. The propagation constant and impedance of a single microstrip line is varied by the presence of the transverse patches (see below). Several models have been implemented for the inductance $L$, e.g., [5], [6], [9]. It was found that [6] provides the best results as the via hole diameter is related to the dimensions of a periodic surface.

The gap between patches or microstrip lines is often modeled by a $\pi$ section of capacitances [10], [11]. For the wide range of EBG parameters investigated, however, this approach was often found out of validity range and produced negative series capacitances. Therefore, the models proposed in [12] and 
[13] were used in which the gap is treated as an admittance inverter with admittance $B_{g}$ centered between two lines of length $\Delta l_{g}$ [13] (Fig. 2a).

In order to incorporate the influence of the transverse patches, a capacitive reactance resulting from the coupling to those patches is normally used, e.g., [5]. It was found, however, that such model produces a number of sharp transitions between pass- and stopbands, which do not appear in solutions of the electromagnetic codes. Therefore, two other approaches have been investigated. First, the gap to the transverse patches can be treated similar to the gap in longitudinal direction. The resulting capacitance $C_{c}$ will then contribute to a wider patch of width $w_{\text {eff }}$ (Fig. 2b). Alternatively, the transverse patches can be modeled as coupled lines according to Fig. 2c. Both approaches have been investigated and were found to produce similar results. Finally, for given cross-section dimensions of the microstrip line, propagation constants and characteristic impedances are calculated via [11] - [13] and include the metallization thickness and frequency dependent terms.

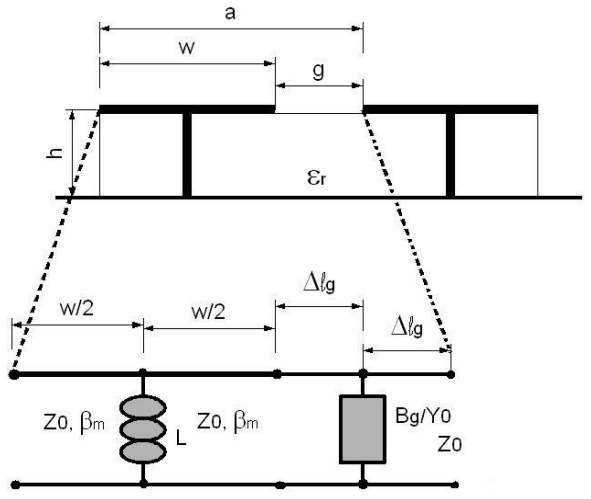

(a) (b)

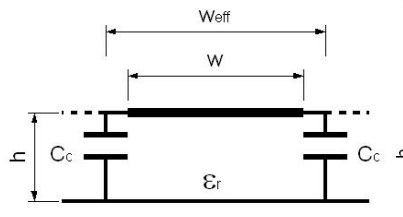

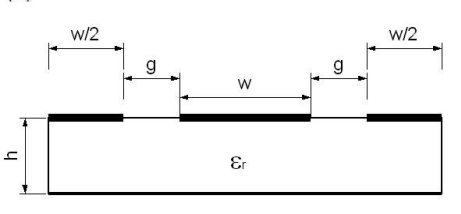

(c)
Figure 2. Transmission-line model of the unit cell in direction of propagation (a), and in transverse direction using an effective width (b) or coupled lines (c).

Once $Z_{0}$ and $\beta_{m}$ are known, the $\mathrm{ABCD}$ matrix of the structure in Fig. 2a is computed and normalized to $Z_{0}$, which is the characteristic impedance on both ends of the unit cell (Fig. $2 \mathrm{a})$. Then voltages and currents at the left of unit cell $n$ of a periodic structure are related to those of cell $n+1$ by

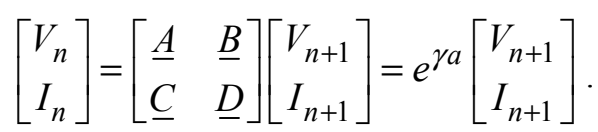

Considering that $\underline{\mathrm{AD}}-\underline{\mathrm{BC}}=1$ for a passive circuit, this results in a quadratic equation for $\lambda$

$$
\lambda^{2}-(\underline{A}+\underline{D}) \lambda+1=0
$$

where

$$
\lambda=R e^{j \phi}=e^{\gamma a}, \quad \gamma=\alpha+j \beta \text {. }
$$

The two complex solutions for $\lambda$ specify the attenuation and propagation constants of the EBG structure [14].

$$
\alpha=\ln (R) / a, \quad \beta=\phi / a
$$

In order to relate the attenuation constant, $\alpha$, for the periodic (infinite) structure to the actual transmission coefficient $\left|S_{21}\right|$ introduced by a finite number of cells, $N$, we employ a relationship introduced in [15] and verified in [16]

$$
\left|S_{21}\right| / d B=-8.686 N \alpha p
$$

where $p=a$ is the length of the unit cell.

\section{RESULTS}

As a direct comparison between a previous and our equivalent-circuit model, the one used in [5] and our approach were implemented in simple Matlab and/or Fortran routines. In order to be able to compare attenuation over a finite number of cells, we added the calculation of the attenuation constant to the model in [5], which follows straightforwardly from [5], and applied equ. (5) for a given number $N$ of cells.

Fig 3a depicts the propagation and attenuation constants calculated from this approach and compares it to results using the model in [5]. Several differences are observed: First, the method in [5] (light curves) predicts a wide passband of varying propagation constant $\beta$ (light dashed line) up to 2.55 $\mathrm{GHz}$, whereas our method clearly indicates a stopband with attenuation constant $\alpha$ (dark dotted line). The unattenuated passband starts at around $2.55 \mathrm{GHz}$ for both methods but is wider in our model (dark solid line) than in that of [5]. Beyond $2.95 \mathrm{GHz}$, the model in [5] predicts a variety of either passbands (light dashed line) or stopbands (light dash-dotted lines). Our model produces a complex propagation constant (dark solid and dotted lines) between $3.6 \mathrm{GHz}$ and $8.1 \mathrm{GHz}$, and propagation continues up to $10 \mathrm{GHz}$.

In order to qualitatively verify the results, an array of $7 x 7$ cells was analyzed by IE3D ${ }^{\circledR}$, Ansoft Designer ${ }^{\circledR}$ and $\mathrm{HFSS}^{\circledR}$. In Fig. $3 b$ the results are compared to our attenuation values and those using the method in [5] as obtained from equ. (5). All three commercial software packages (although their differences are surprisingly large) clearly predict a stopband up to about $2.6 \mathrm{GHz}$ followed by a passband much wider than predicted by the method in [5] (light long-dashed line). The end of this first passband falls very close to our prediction (dark solid line). Towards higher frequencies, the commercial packages predict continuing propagation with some attenuation. Although the agreement in this higher frequency range is not as good as towards lower frequencies, our model correctly predicts the first stopband, the first passband and the continued propagation thereafter. In comparison, the model in [5] fails to predict the first stopband, produces a narrower first passband directly followed by a narrow stopband and another narrow stopband at $7.5 \mathrm{GHz}$. None of these stopbands are qualitatively verified by any of the three commercial electromagnetic codes. We therefore conclude that the results obtained with our model, as simple as it is, are in good agreement with other, more complicated techniques and that it is superior to that of [5]. 

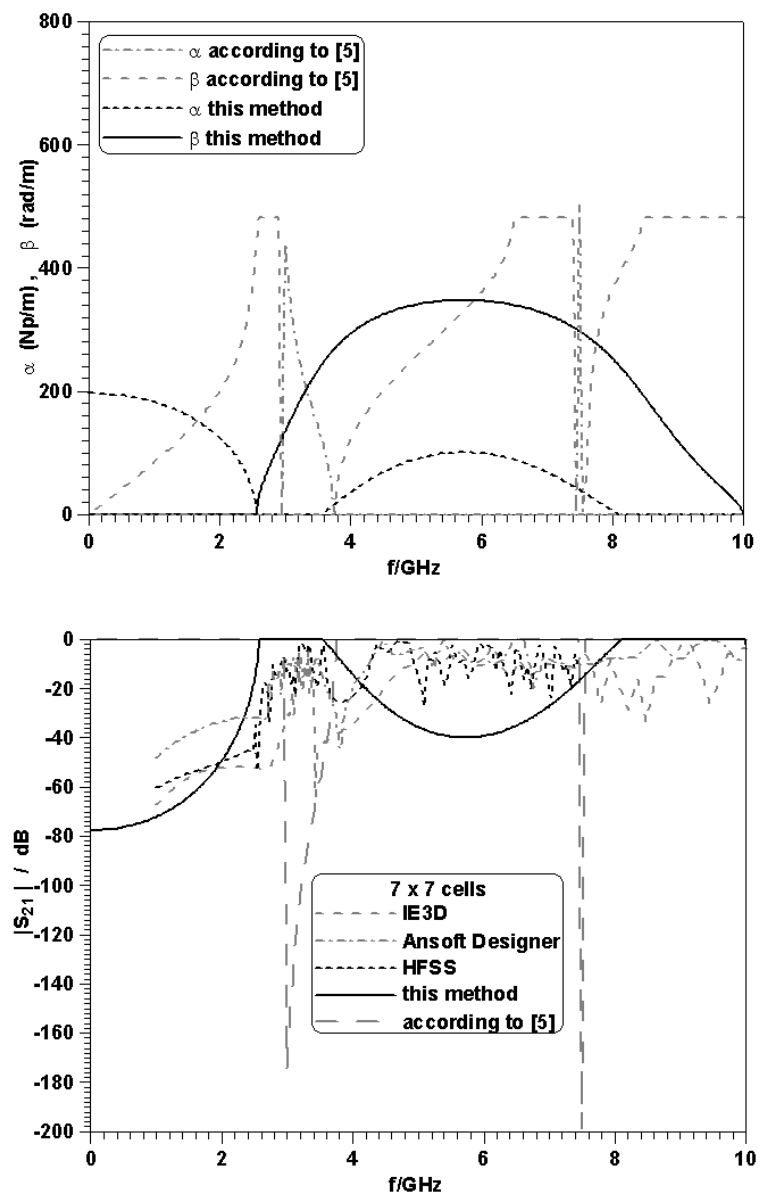

(a)

(b)

Figure 3. (a) Comparison of propagation characteristics between this model and that of [5]; (b) transmission coefficient of a 7x7 cell structure and comparison between this model,that of [5] and three commercial field solvers. Dimensions (c.f. Fig. 1): $\mathrm{w}=6.0 \mathrm{~mm}, \mathrm{~g}=0.5 \mathrm{~mm}, \mathrm{a}=6.5 \mathrm{~mm}, \mathrm{~d}=1.0 \mathrm{~mm}, \mathrm{~h}=2.54 \mathrm{~mm}, \varepsilon_{\mathrm{r}}=10.2$.

Fig. 4a shows the propagation characteristics of an EBG structure with wider stopband performance. In comparison with the configuration in Fig. 3, the permittivity is reduced and the patches as well as the substrate height increased. For a $7 \times 7$ array, our $\left|S_{21}\right|$ data is compared to results from IE3D ${ }^{\circledR}$ and Ansoft Designer ${ }^{\circledR}$ in Fig. 4b, and good agreement is observed. The passband is correctly predicted (although somewhat wider), and the basic shape of the curve is reproduced.

The final example uses dimensions appropriate for fabrication in LTCC technology. Fig. 5a presents the propagation characteristics and Fig. 5b a comparison of transmission behavior with $\mathrm{HFSS}^{\circledR}$. Good agreement is again observed, especially for the start of the passband.

Note that the ripple in the passbands of the field solvers' calculations is attributed to reflections within the small number of cells. They should weaken once a higher number of cells is considered, which was not possible in this investigation due to memory limitations.

\section{CONCLUSIONS}

The simple and improved equivalent-circuit model presents a viable approach for the fast analysis and design of EBG structures formed by via-holed patches. The first stop- and passbands are correctly predicted over a broad frequency range as verified by comparison with independent numerical codes. Moreover, the complex propagation constant towards higher frequencies is an adequate representation of behavior as compared to field solvers. Hence this technique represents an improvement over known similar models. Although the derived propagation characteristics apply only in the two orthogonal directions of the EBG structure, other directions of propagation can be analyzed using superposition.

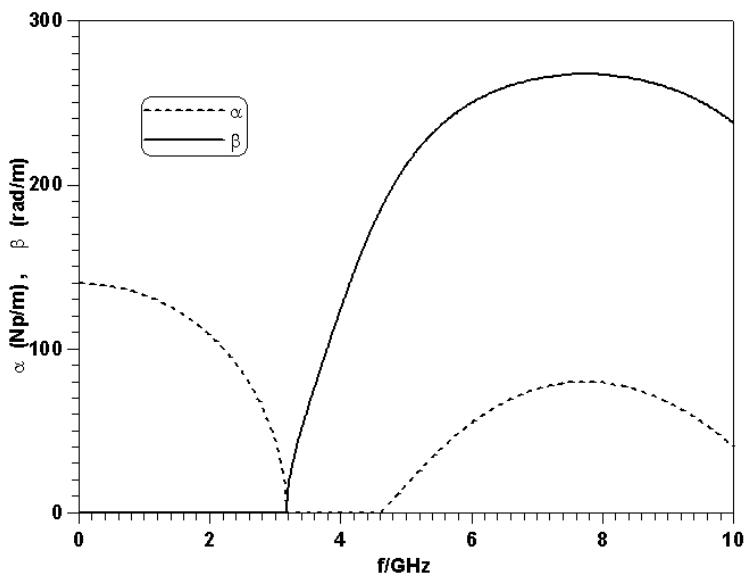

(a)

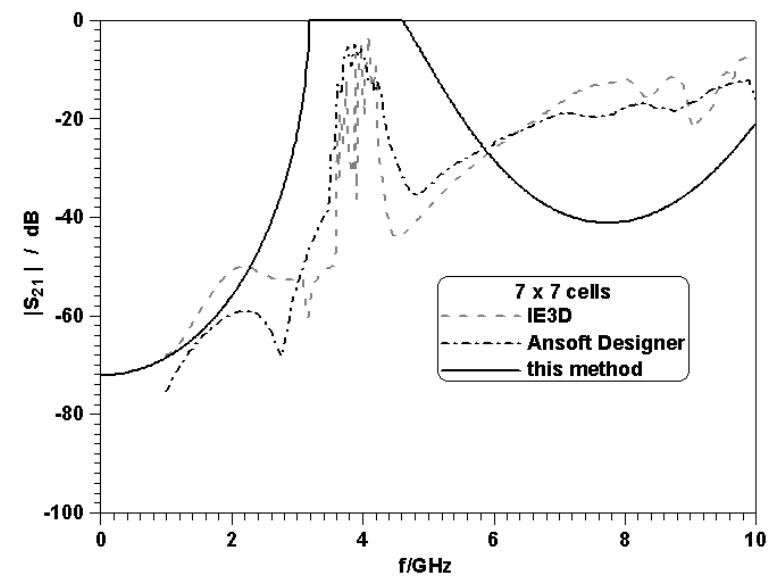

(b)

Figure 4. Propagation characteristics (a); transmission coefficient (b) comparing the results of this method (solid line with those of IE3D (dashed line) and Ansoft Designer (dash-dotted line). Dimensions (c.f. Fig. 1): $\mathrm{w}=8.0 \mathrm{~mm}, \mathrm{~g}=0.5 \mathrm{~mm}, \mathrm{a}=8.5 \mathrm{~mm}, \mathrm{~d}=1.0 \mathrm{~mm}, \mathrm{~h}=3.175 \mathrm{~mm}, \varepsilon_{\mathrm{r}}=2.35$.

\section{REFERENCES}

[1] G. Eason, B. Noble, and I. N. Sneddon, V. Radisic, Y. Qian, R. Coccioli and T. Itoh, "Novel 2-D photonic band-gap structures for microstrip lines," IEEE Microwave Guided Wave Lett., Vol. 8, pp. 69-71, Feb. 1998.

[2] H. Mosallaei and Y. Rahmat-Samii, "Broadband characterization of complex periodic EBG structures: An FDTD/prony technique based on the split-flied approach", Electromagnetics, Vol. 23, pp. 135-151, Feb. 2003.

[3] L. Zhang and N.G. Alexopoulos, "Finite-element-based techniques for the modeling of PBG materials," Electromagnetics, Vol. 19, No. 3, pp. 225-240, May-June 1999. 

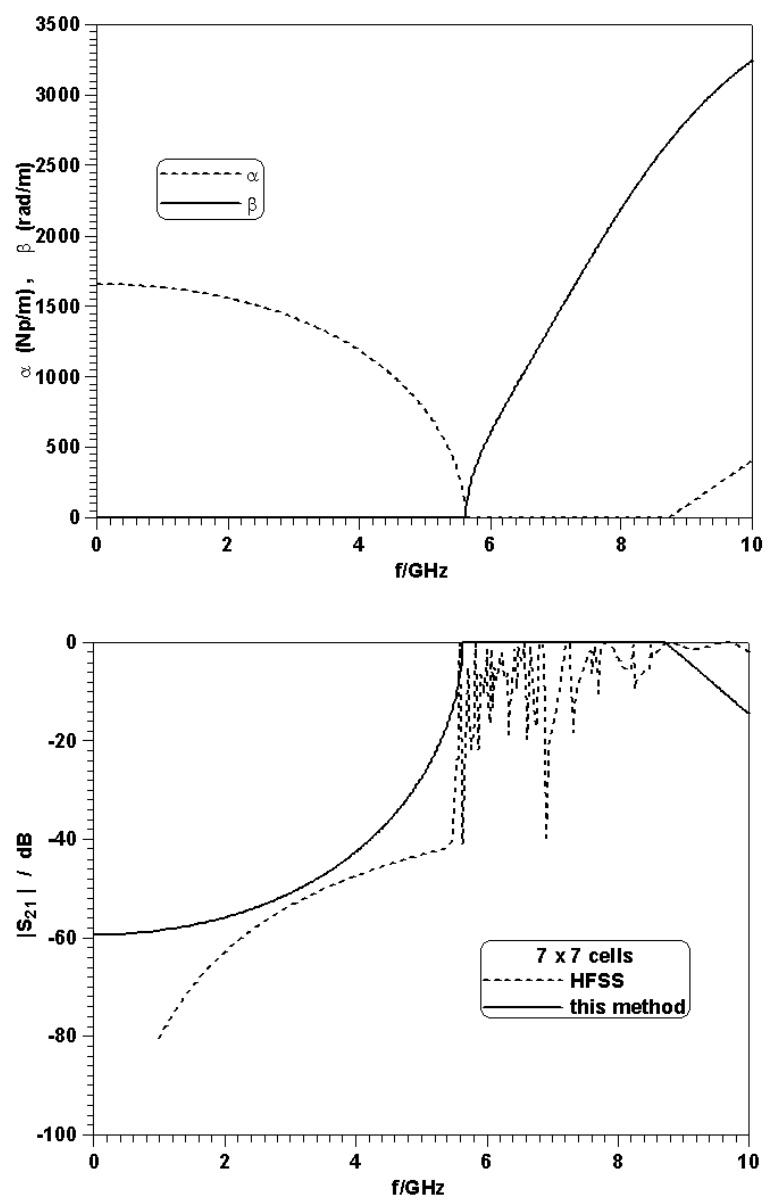

(b)

Figure 5. Propagation characteristics (a); transmission coefficient (b) comparing the results of this method (solid line with those of HFSS (dotted line). Dimensions (c.f. Fig. 1): $w=0.54 \mathrm{~mm}, \mathrm{~g}=0.05 \mathrm{~mm}, \mathrm{a}=0.59 \mathrm{~mm}, \mathrm{~d}=0.05 \mathrm{~mm}$, $\mathrm{h}=0.8 \mathrm{~mm}, \varepsilon_{\mathrm{r}}=58$.
[4] Y. Toyota, A.E. Engin, T.H. Kim and M. Swaminathan, "Stopband analysis using dispersion diagram for two-dimensional electromagnetic bandgap structures in printed circuit boards," IEEE Microwave Wireless Comp. Lett., Vol. 16, pp. 645-647, Dec. 2006.

[5] M. Rahman and M.A. Stuchly, "Transmission line - periodic circuit representation of planar microwave photonic bandgap structures," Microwave Opt. Techn. Lett., Vol. 30, No. 1, pp. 15-19, July 2001.

[6] S.D. Rogers, "Electromagnetic-bandgap layers for broad-band suppression of TEM modes in power planes," IEEE Trans. Microwave Theory Tech., Vol. 53, pp. 2495-2505, Aug. 2005.

[7] S. Clavijo, R.E. Díaz and W.E. McKinzie, III, "Design methodology for Sievenpiper high-impedance surfaces: An artificial magnetic conductor for positive gain electrically small antennas," IEEE Trans. Antennas Propagat., Vol. 51, pp. 2678-2690, Oct. 2003.

[8] D.F. Sievenpiper, L. Zhang, F.J. Broas, N.G. Alexopoulos and E. Yablonovitch, "High-impedance electromagnetic surfaces with a forbidden frequency band," IEEE Trans. Microwave Theory Tech., Vol. 47, pp. 2059-2074, Nov. 1999.

[9] M.E. Goldfarb and R.A. Pucel, "Modeling via hole grounds in microstrip", IEEE Microwave Guided Wave Lett., Vol. 1, pp. 135-137, June 1991.

[10] P. Benedek and P. Silvester, "Equivalent capacitances for microstrip gaps and steps", IEEE Trans. Microwave Theory Tech, Vol. 20, pp. 729733, Nov. 1972.

[11] R.K. Hoffmann, Handbook of Microwave Integrated Circuits, Artech House, Boston, 1987.

[12] E. Hammerstad and O. Jensen, "Accurate models for microstrip computeraided design", in 1980 IEEE MTT-S Int. Microwave Symp. Dig., pp. 407-409, 1980.

[13] E. Hammerstad, "Computer-aided design of microstrip couplers with accurate discontinuity models", in 1981 IEEE MTT-S Int. Microwave Symp. Dig., pp. 54-56, 1981.

[14] S. Amari, R. Vahldieck, J. Bornemann and P. Leuchtmann, "Spectrum of corrugated and periodically loaded waveguides from classical matrix eigenvalues", IEEE Trans. Microwave Theory Tech., Vol. 48, pp. 453460, Mar. 2000.

[15] S. Amari, J. Bornemann and R. Vahldieck, "Simple rules for truncation of periodic structures to achieve a prescribed bandgap attenuation level", in XXVIth URSI General Assembly Abstracts, p. 39, Toronto, Canada, Aug. 1999.

[16] J. Bornemann, S. Amari, and R. Vahldieck, "A flexible CIET analysis for the design of on-axis circular waveguide components", in Proc. 2003 Asia Pacific Microwave Conf., pp. 1467-1470, Seoul, Korea, Nov. 2003. 